\title{
Capturing Velocity Gradients and Particle Rotation Rates in Turbulence
}

\author{
Leonhard A. Leppin $\oplus^{1,2,3}$ and Michael Wilczek $\oplus^{1,2, *}$ \\ ${ }^{1}$ Max Planck Institute for Dynamics and Self-Organization (MPI DS), Am Faßberg 17, 37077 Göttingen, Germany \\ ${ }^{2}$ Faculty of Physics, Georg-August-Universität Göttingen, Friedrich-Hund-Platz 1, 37077 Göttingen, Germany \\ ${ }^{3}$ Max Planck Institute for Plasma Physics, Boltzmannstraße 2, 85748 Garching, Germany
}

(Received 6 May 2020; revised 15 September 2020; accepted 26 September 2020; published 25 November 2020)

\begin{abstract}
Turbulent fluid flows exhibit a complex small-scale structure with frequently occurring extreme velocity gradients. Particles probing such swirling and straining regions respond with an intricate shape-dependent orientational dynamics, which sensitively depends on the particle history. Here, we systematically develop a reduced-order model for the small-scale dynamics of turbulence, which captures the velocity gradient statistics along particle paths. An analysis of the resulting stochastic dynamical system allows pinpointing the emergence of non-Gaussian statistics and nontrivial temporal correlations of vorticity and strain, as previously reported from experiments and simulations. Based on these insights, we use our model to predict the orientational statistics of anisotropic particles in turbulence, enabling a host of modeling applications for complex particulate flows.
\end{abstract}

DOI: 10.1103/PhysRevLett.125.224501

Turbulent flows show complex dynamics with a wide range of dynamically active scales [1-3], which play an important role for the dispersal of pollutants and aerosols in the atmosphere [4,5], the transport of microorganisms in the ocean [6-10], as well as the mixing of reactants in turbulent combustion [11,12]. The smallest turbulent scales, which are essentially independent of the boundaries and anisotropies of the large-scale flow [1], have a profound impact on the dynamics and collision rates of small suspended particles, like plankton in the ocean [6-10], as well as droplets and ice crystals in clouds [13-16]. Even in the simplest case of very small, neutrally buoyant particles, which passively follow the velocity field, highly nontrivial shape-dependent rotational motion has been observed [17-22]. Theoretically, this intricate dynamics is not well understood.

The spinning and tumbling of particles immersed in a turbulent flow are determined by the complex interplay of particle shape and the small-scale structure of the turbulent flow field, as encoded in the gradients of the velocity field $A_{i j}=\partial u_{i} / \partial x_{j}$ [23]. Because particle rotations are very sensitive to various small-scale features of turbulence such as non-Gaussian fluctuations, the local flow topology and, most importantly, the temporal correlation of strain and vorticity along Lagrangian trajectories, capturing this

Published by the American Physical Society under the terms of the Creative Commons Attribution 4.0 International license. Further distribution of this work must maintain attribution to the author(s) and the published article's title, journal citation, and DOI. Open access publication funded by the Max Planck Society. complex motion with theoretically insightful reduced-order models for turbulence so far remained elusive. The challenges in predicting these aspects of turbulent velocity gradients ultimately arise from the nonlinear, nonlocal, and dissipative dynamics of the governing Navier-Stokes equations.

Over the past years, a variety of reduced-order models for the velocity gradient statistics based on stochastic differential equations (SDEs) has been developed [23-29]. In these models, the effects of nonlocal pressure and viscous diffusion result in unclosed terms, to which diverse closure techniques have been applied. Closure theories range from models based on prescribed log-normal dissipation rates [24], the coarse-grained velocity gradient as perceived by a tetrad of tracer particles $[25,26]$, or the deformation of fluid elements [27] to functional closures based on Gaussian random fields [28], as well as combinations of these approaches [29]. The most advanced reduced-order SDE models successfully reproduce many of the characteristic geometric and statistical properties of the turbulent small scales [28-32]. However, all current models struggle to capture important aspects of the temporal correlation of strain rate and rotation rate, which, in particular, leads to poor predictions for the orientational dynamics of particles immersed in turbulent flows.

Here, we develop a minimal model for the velocity gradients in turbulence, which enables profound theoretical insights. Starting from an exact statistical evolution equation, we systematically constrain its structure based on tensor function representation theory. By using an ensemble approach, we construct a physically consistent model that complies with important homogeneity constraints of turbulent fields. Based on an analysis of the associated 
Fokker-Planck equation, we establish a clear interpretation of its nonlinear dynamics. Specifically, we identify the dynamical mechanisms which control the degree of nonGaussianity and temporal correlations of vorticity and strain. We test our predictions against high-resolution simulation results of fully developed turbulence and show that our model captures the temporal autocorrelations of rotation rate and strain rate. Coupled to the equations for the orientation dynamics of ellipsoidal particles, our model, furthermore, accurately reproduces the tumbling and spinning rates of particles in turbulent flows.

The evolution equation for velocity gradients is obtained by taking the gradient of the Navier-Stokes equation. Along a tracer particle, the velocity gradient changes according to the local self-amplification of velocity gradients, nonlocal pressure contributions, viscous diffusion, and external forces [23]. The foundation of our model is an exact, unclosed SDE for the one-point statistics of homogeneous isotropic turbulence, which statistically captures these various contributions to the dynamics. Using stochastic calculus, one can derive this SDE from the Navier-Stokes equations [28]. It takes the form

$$
d \boldsymbol{A}=\left(-\widetilde{\boldsymbol{A}^{2}}-\langle\widetilde{\boldsymbol{H}} \mid \boldsymbol{A}\rangle+\langle\nu \Delta \boldsymbol{A} \mid \boldsymbol{A}\rangle\right) d t+d \boldsymbol{F} .
$$

Here, $\boldsymbol{A}$ can be interpreted as the stochastic process corresponding to the velocity gradient field at the position of a fluid particle. The tilde denotes the traceless part of the tensor, e.g., $\widetilde{A^{2}}=\left[\boldsymbol{A}^{2}-\frac{1}{3} \operatorname{Tr}\left(\boldsymbol{A}^{2}\right) \boldsymbol{I}\right]$. The first term on the right-hand side, which appears in closed form, captures the nonlinear local self-amplification of the velocity gradient. It includes the local isotropic part of the pressure Hessian $H_{i j}=\left(\partial^{2} p\right) /\left(\partial x_{i} \partial x_{j}\right)$, which is obtained from the pressure Poisson equation $\Delta p=\operatorname{Tr}(\boldsymbol{H})=-\operatorname{Tr}\left(\boldsymbol{A}^{2}\right)$ [33]. Unclosed terms, which contain information beyond the local single-point statistics, appear in the form of conditional averages, i.e., as averaged fields conditional on a given configuration of the velocity gradient at the same position. The conditional average $\langle\widetilde{\boldsymbol{H}} \mid \boldsymbol{A}\rangle$ contains information about the mean nonlocal, deviatoric part of the pressure Hessian given a velocity gradient configuration $\boldsymbol{A}$, which a priori depends on the full flow field due to the pressure Poisson equation. The conditional Laplacian $\langle\nu \Delta \boldsymbol{A} \mid \boldsymbol{A}\rangle$ encodes viscous effects in the velocity gradient tensor evolution. The term $d \boldsymbol{F}$ is a Gaussian, temporally delta-correlated tensorial forcing, which is consistent with isotropy, homogeneity, and incompressibility $\operatorname{Tr}(\boldsymbol{A})=0$. It naturally arises when considering stochastically forced turbulence.

To close the conditional mean pressure Hessian and the conditional mean Laplacian terms, we express them as isotropic tensor-valued functions of the symmetric and antisymmetric part of the velocity gradient, the strain rate $S$, and rotation rate $\boldsymbol{W}$, respectively. Using tensor function representation theory, one can derive a complete and irreducible representation in terms of a small number of tensorial terms [34-39]. The individual tensorial terms are comprised of combinations of $\boldsymbol{S}$ and $\boldsymbol{W}$, with coefficient functions that depend on isotropic invariants of $S$ and $\boldsymbol{W}$. The conditional mean traceless, symmetric pressure Hessian, for example, can be expressed as a linear combination of seven tensorial terms with appropriate coefficient functions (cf. Supplemental Material [40], Sec. I). Previous studies [28,47] showed that the most important features of the dynamics can already be captured by retaining terms up to the lowest possible order (i.e., up to second order in the pressure Hessian and up to first order in the viscous term) with constant coefficients. To enable analytical insights, we therefore truncate the general tensorial expansion and consider the closure

$$
\begin{aligned}
\langle\widetilde{\boldsymbol{H}} \mid \boldsymbol{A}\rangle & =\alpha \widetilde{\boldsymbol{S}^{2}}+\beta \widetilde{\boldsymbol{W}^{2}}+\gamma(\boldsymbol{S} \boldsymbol{W}-\boldsymbol{W S})+\delta \boldsymbol{S}, \\
\langle\nu \Delta \boldsymbol{A} \mid \boldsymbol{A}\rangle & =\xi \boldsymbol{A} .
\end{aligned}
$$

This expression still contains five scalar parameters, which need to be further constrained. A general limitation of single-point closures is that they lack the possibility to include physical constraints that depend on information from the full field. For homogeneous turbulence, for example, the velocity gradient field fulfills the Betchov constraints [48] $\left\langle\operatorname{Tr}\left(\boldsymbol{A}^{2}\right)\right\rangle=0$ and $\left\langle\operatorname{Tr}\left(\boldsymbol{A}^{3}\right)\right\rangle=0$, which encode the balance of enstrophy and dissipation, as well as of their production. So far, velocity gradient models need careful calibration to fulfill these constraints [29]. An intriguing alternative to achieve a model that is physically consistent with homogeneous turbulence is to consider an ensemble of Lagrangian fluid elements that sample the full velocity gradient field. We then achieve consistency with the Betchov constraints by identifying spatial averages over the field with ensemble averages over the Lagrangian fluid elements. This can be used to derive analytical expressions from (1) for two of the parameters, which allows us to constrain our closure (2) and (3). One additional parameter can be fixed by nondimensionalizing the velocity gradient model with the Kolmogorov timescale $\tau_{\eta}$, which implies $\left\langle\operatorname{Tr}\left(\boldsymbol{S}^{2}\right)\right\rangle=1 / 2$. We choose to constrain the parameters $\beta$, $\delta$, and $\xi$ and obtain them as functions of ensemble-averaged scalar invariants of the velocity gradient and the remaining parameters. Their explicit form and derivation is given in the Supplemental Material, Sec. II [40]. Thereby, the parameter space is reduced by three dimensions, and Betchov's homogeneity constraints are fulfilled by design. Besides the forcing amplitude, which we fix for the following considerations (see Supplemental Material [40], Sec. III for the impact of the forcing amplitude), this leaves two free parameters: $\alpha$ and $\gamma$.

The impact of these free parameters on the nonlinear dynamics of the velocity gradient model can be revealed from the Fokker-Planck equation (FPE) corresponding 

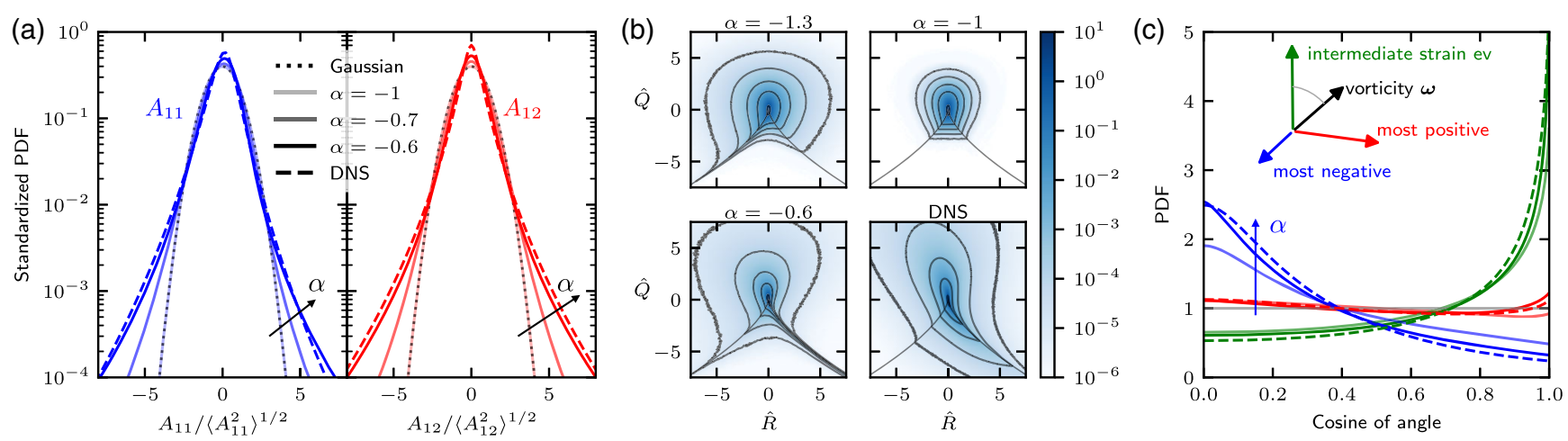

FIG. 1. Controlling single-time statistics with the strength of the strain self-amplification $\alpha$. (a) $\alpha$ controls the departure from Gaussianity of the model statistics: Standardized PDFs of the longitudinal $\left(A_{11}\right)$ and transverse $\left(A_{12}\right)$ velocity gradient components for different values of $\alpha$. (b) $\alpha$ determines the probability of different flow topologies: Joint PDF of the standardized isotropic invariants $\hat{R}=-\operatorname{Tr}\left(\mathbf{A}^{3}\right) /\left[3\left\langle\operatorname{Tr}\left(\mathbf{S}^{2}\right)\right\rangle^{3 / 2}\right]$ and $\hat{Q}=-\operatorname{Tr}\left(\mathbf{A}^{2}\right) /\left[2\left\langle\operatorname{Tr}\left(\mathbf{S}^{2}\right)\right\rangle\right]$ for different values of $\alpha$. DNS results in lower right panel. The Vieillefosse line is indicated in gray. (c) Alignment of principal strain axes and vorticity increases with strain self-amplification: PDFs of the cosine of the angle between the vorticity vector and the three eigenvectors of the strain-rate tensor for the same values of $\alpha$ as in (a).

to (1), which governs the evolution of the full probability density function (PDF) $f(\boldsymbol{A} ; t)$ of the velocity gradient tensor (implied summation),

$\frac{\partial}{\partial t} f=-\frac{\partial}{\partial A_{i j}}\left[\left(N_{i j}+L_{i j}\right) f\right]+\frac{1}{2} Q_{i j k l}(\mathbf{0}) \frac{\partial}{\partial A_{i k}} \frac{\partial}{\partial A_{j l}} f$.

Here, $Q_{i j k l}(\mathbf{0})$ denotes the forcing covariance, and the nonlinear and linear drift terms are given by

$$
\begin{aligned}
& \boldsymbol{N}=-(1+\alpha) \widetilde{\boldsymbol{S}^{2}}-(1+\beta) \widetilde{\boldsymbol{W}^{2}}-(1+\gamma) \boldsymbol{S W}-(1-\gamma) \boldsymbol{W} \boldsymbol{S} \\
& \boldsymbol{L}=-\delta \boldsymbol{S}+\xi \boldsymbol{A}
\end{aligned}
$$

The parameter $\alpha$ controls the strength of the strain selfamplification in the velocity gradient dynamics. For a vanishing self-amplification $(\alpha=-1)$ and parameters determined such that the Betchov constraints are fulfilled, we find that (4) has an exact Gaussian solution (cf. Supplemental Material [40], Sec. IV.1). Remarkably, even in this case, the FPE contains nonlinear drift terms. We demonstrate below that the strength of strain selfamplification controls departures from Gaussianity as well as important features of the small-scale topology of the predicted velocity gradient statistics. Further analysis of the FPE shows that the single-time statistics is independent of the parameter $\gamma$ for isotropic turbulence. In this case, the velocity gradient PDF is a function of the tensor invariants only, and one can readily calculate that $\partial\left[\gamma\left(S_{i k} W_{k j}-\right.\right.$ $\left.\left.W_{i k} S_{k j}\right) f(\mathbf{A})\right] / \partial A_{i j}=0$ (cf. Supplemental Material [40], Sec. IV.2). This result is related to a recently reported gauge symmetry of the pressure Hessian [49]. However, we show below that, for the two-time statistics, and, in particular, the autocorrelations of vorticity and strain, the $\gamma$-term turns out to be crucial.
To determine appropriate values for the free parameters, we perform parameter scans and compare our model results with velocity gradient statistics obtained from direct numerical simulations (DNS) of the Navier-Stokes equation. We analyzed a simulation of homogeneous isotropic turbulence with $2048^{3}$ grid points on a periodic domain at a Taylor-scale Reynolds number of $R_{\lambda} \approx 509$ with a smallscale resolution of $k_{\max } \eta \approx 1.5$ ( $k_{\max }$ is the largest resolved wave number, and $\eta$ is the Kolmogorov scale). For the data analysis, 25 snapshots spanning ca. four integral timescales are taken into account. For the parameter scans, we numerically solve (1) using the EulerMaruyama method [50] with a time step of $\Delta t=0.0002$. Convergence checks with varying time steps were also performed. For all simulations shown here, we have integrated an ensemble of $10^{5}$ Gaussian initial conditions for $5 \times 10^{6}$ time steps, which corresponds to $1000 \tau_{\eta}$ in physical time, after an initial transient of $100 \tau_{\eta}$ (cf. Supplemental Material [40], Sec. V). Initial simulations of (1) revealed the occurrence of rare rogue trajectories exploring far-out regions of the phase space, which leads to nonconvergent statistics and may introduce numerical instabilities in the determination of our parameters. We identified the second-order truncation of the unclosed terms as the origin of this shortcoming, which can be remedied by including a nonlinear damping term (cf. Supplemental Material [40], Sec. VI). The auxiliary term $\epsilon \boldsymbol{A}$, which is added to (3), is constructed to damp trajectories that diverge far from the ensemble mean and is negligibly small for the major, dynamically most relevant part of phase space; we set $\epsilon=-10^{-8}\left\{\left[\operatorname{Tr}\left(\boldsymbol{W}^{2}\right)+1 / 2\right]^{4}+\left[\operatorname{Tr}\left(\boldsymbol{S}^{2}\right)-1 / 2\right]^{4}\right\}$.

Figure 1(a) illustrates how the strength of strain selfamplification controls the departure from Gaussianity of the predicted velocity gradient statistics. As $\alpha$ deviates from -1 , the single-component PDFs become non-Gaussian with increasingly heavy tails. For $\alpha=-0.6$, the 
standardized PDFs of the velocity gradient components of our model agree well with DNS results within 7 standard deviations [cf. Fig. 1(a)]. The match is particularly good for the transverse components, for which our model captures the vanishing skewness and closely matches the DNS kurtosis of $\left\langle A_{12}^{4}\right\rangle /\left\langle A_{12}^{2}\right\rangle^{2} \approx 14.34$ to within $1 \%$ (model: 14.32). For the longitudinal components, our model underpredicts the skewness $\left\langle A_{11}^{3}\right\rangle /\left\langle A_{11}^{2}\right\rangle^{3 / 2}$ (model: -0.42 ; DNS: -0.61), consistent with other recent models [29], and slightly overpredicts the kurtosis (model: 11.46; DNS: 9.2).

Furthermore, the strain self-amplification determines the probability of different flow topologies, as encoded in the invariants $Q=-\operatorname{Tr}\left(A^{2}\right) / 2$ and $R=-\operatorname{Tr}\left(A^{3}\right) / 3$, which capture the competition between enstrophy and dissipation, as well as between their production. In the $R-Q$ plane, the Vieillefosse line, i.e., the zero crossing of the discriminant of $\boldsymbol{A}$ given by (27/4) $R^{2}+Q^{3}=0$, plays an important role, as it separates the upper region with complex eigenvalues of $\boldsymbol{A}$ from the lower region with purely real eigenvalues. Figure 1(b) shows the joint PDF of the standardized invariants $\hat{Q}$ and $\hat{R}$ for different values of $\alpha$ and from DNS. As $\alpha$ is tuned from -1.3 to -0.6 , the joint PDF first extends along the left part of the Vieillefosse line, becomes symmetric for Gaussian statistics $(\alpha=-1)$, and finally extends along the right part of the Vieillefosse line. This corresponds to a shift of probability from flow regions with two compressive principal strain directions to regions with two extensional principal strain directions [51]. For $\alpha=-0.6$, our model qualitatively captures the shape of the PDF as observed in DNS (lower right panel) and experiments [23], although the probability of velocity gradient configurations along the right part of the Vieillefosse line is underestimated. Nonetheless, since our model inherently fulfills the Betchov constraints, the mean of our model $\hat{R}-\hat{Q}$ PDF lies accurately at $\langle\hat{R}\rangle=\langle\hat{Q}\rangle=0$ for all values of $\alpha$.

Strain self-amplification also impacts another important aspect of the small-scale topology: the alignment between the vorticity vector and the principal strain-rate axes. Figure 1(c) shows the PDFs of the cosine of the angle between the vorticity vector and the three eigenvectors of the strain-rate tensor. Our model (with $\alpha=-0.6$ ) accurately captures the alignment of the vorticity with all three eigenvectors. In particular, it captures the well-known preferential alignment of the vorticity with the eigenvector to the intermediate eigenvalue $[23,52,53]$. The alignment strength decreases with decreasing self-amplification, and for $\alpha=-1$, when the strain self-amplification vanishes and the model statistics are Gaussian, as expected, no preferential alignment is observed.

While we showed analytically that the $\gamma$-term has no effect on the single-time statistics, it determines temporal correlations of the velocity gradients. This can be rationalized from the fact that $\gamma(\boldsymbol{S W}-\boldsymbol{W} \boldsymbol{S})$ essentially rotates the strain eigenframe about the axis given by the
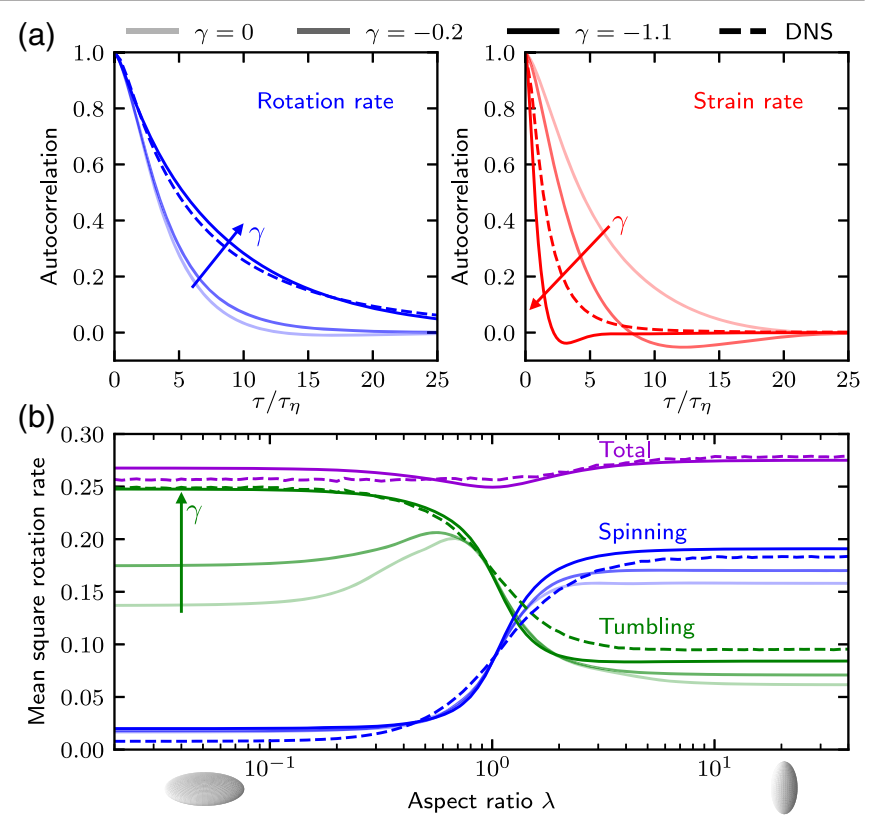

FIG. 2. Controlling temporal correlations and particle rotation rates with $\gamma$. (a) Temporal autocorrelation of rotation rate (blue) and strain rate (red) for different values of $\gamma$ and DNS. (b) Mean square rotation rates of anisotropic particles as a function of the particles' aspect ratio as predicted by our model and DNS, nondimensionalized by the Kolmogorov timescale. The tumbling rates (green) and spinning rates (blue) are plotted for three different values of $\gamma$ corresponding to (a). The total rotation rate (sum of spinning and tumbling) is plotted for $\gamma=-1.1$ in violet.

vorticity vector with a rotation rate proportional to the vorticity magnitude [28]. This directly impacts the temporal correlation of velocity gradients and allows one to precisely control them. Figure 2(a) compares temporal correlations of velocity gradients $\left\langle C_{i j}(t)\right.$ $\left.C_{i j}(t+\tau)\right\rangle / \sqrt{\left\langle C_{m n}(t)^{2}\right\rangle\left\langle C_{p q}(t+\tau)^{2}\right\rangle}$ (implied summation) of our model to DNS results, where $C_{i j}=S_{i j}$ or $W_{i j}$. For the DNS results, the simulation was continued with $10^{6}$ Lagrangian tracer particles, and we collected data from the statistically stationary state. For $\gamma=-1.1$, our model matches the vorticity autocorrelation very well. Importantly, it also captures the previously observed [54] shorter correlation time of the rate of strain compared to the rate of rotation, although differences occur in the shape of the correlation function. These results show, in particular, that the rotation of the strain eigenframe as encoded by the $\gamma$-term is responsible for a decrease of the correlation time of the strain rate and an increase of the rotation-rate correlation time.

Having established a model that captures the different temporal correlations of strain and vorticity along with the central non-Gaussian features of small-scale turbulence, we can use it to predict the tumbling and spinning rates of Lagrangian particles. To this end, we couple our model to Jeffery's equation [55], which describes the orientational 
dynamics of axisymmetric ellipsoidal particles (implied summation),

$$
\frac{d}{d t} p_{i}=W_{i j} p_{j}+\frac{\lambda^{2}-1}{\lambda^{2}+1}\left(S_{i j} p_{j}-p_{i} p_{k} S_{k l} p_{l}\right) .
$$

Here, the unit vector $\boldsymbol{p}$ denotes a particle's symmetry axis, and $\lambda$ is the particle's aspect ratio, i.e., the ratio of the length along the symmetry axis to the length perpendicular to it. The rotation of an axisymmetric particle can be decomposed into spinning (rotation around the symmetry axis) and tumbling (rotations around axes perpendicular to the symmetry axis) [17], with the squared spinning rate $\left(\frac{1}{2} \boldsymbol{\omega} \cdot \boldsymbol{p}\right)^{2}$, where $\boldsymbol{\omega}$ is the vorticity, and the squared tumbling rate $\dot{p}_{i} \dot{p}_{i}$. In Fig. 2(b), the nondimensionalized mean square tumbling and spinning rates as predicted by our model are shown for different values of $\gamma$ as a function of the particles' aspect ratio. Figure 2(b) shows that especially the tumbling rates of disklike particles increase when the temporal correlations are modified by increasing the magnitude of the coefficient $\gamma$. When our model exhibits the most realistic correlation times, i.e., for $\gamma=-1.1$, the particle rotation rates predicted by our model agree very well with the ones observed in our DNS and literature $[17,20]$ for the full range of particle shapes. In particular, our model predicts the high tumbling rates of disklike particles observed in DNS and experiments. The comparison of the results for different values of $\gamma$ in Figs. 2(a) and 2(b) indicates that the realistic autocorrelation times of our model are crucial for an accurate prediction of tumbling rates of suspended particles.

In summary, we have analyzed the dynamics and statistics of velocity gradients in turbulence in the framework of a minimal, physically consistent reduced-order model. Our combined analytical and computational analysis showed that strain self-amplification controls the non-Gaussianity as well as the small-scale topology of the velocity gradient dynamics and identified the rotation of the strain eigenvectors by the vorticity as the major factor in determining the temporal correlations of velocity gradients. As a result, we obtained a reduced-order model for the small scales of turbulence that captures the different correlation times of strain and vorticity in turbulence. We showed that the reduced-order model can be used to accurately predict the orientational statistics of suspended anisotropic particles, enabling a host of modeling applications for complex particulate flows.

Based on tensor function representation theory and the systematic implementation of physical constraints, our closure approach explicitly uncovers the general tensorial structure of the unclosed terms, which also provides a firm foundation for future advancements. For example, we expect that the inclusion of higher-order terms and coefficient functions that depend on velocity gradient tensor invariants will lead to further quantitative improvement. Machine learning approaches [56] could turn out to be instrumental in achieving such improved parameterizations.

We thank José-Agustín Arguedas-Leiva, Tobias Bätge, Cristian Lalescu, and Bérenger Bramas for scientific computing support and the provision of the DNS data. Valuable feedback on the manuscript from Lukas Bentkamp and Maurizio Carbone is gratefully acknowledged. The authors gratefully acknowledge the Gauss Centre for Supercomputing e.V. for funding this project by providing computing time on the GCS Supercomputer SuperMUC at Leibniz Supercomputing Centre. Computational resources from the Max Planck Computing and Data Facility and support by the Max Planck Society are gratefully acknowledged.

*michael.wilczek@ds.mpg.de

[1] S. B. Pope, Turbulent Flows (Cambridge University Press, Cambridge, New York, 2000), https://doi.org/10.1017/ CBO9780511840531.

[2] U. Frisch, Turbulence: The Legacy of A.N. Kolmogorov (Cambridge University Press, Cambridge, England, 1995), https://doi.org/10.1017/CBO9781139170666.

[3] A. Tsinober, An Informal Conceptual Introduction to Turbulence, 2nd ed., Fluid Mechanics and Its Applications Vol. 92 (Springer, Dordrecht, 2009), https://doi.org/10 .1007/978-90-481-3174-7.

[4] H. J. S. Fernando, D. Zajic, S. Di Sabatino, R. Dimitrova, B. Hedquist, and A. Dallman, Phys. Fluids 22, 051301 (2010).

[5] J. Chun, D. L. Koch, S. L. Rani, A. Ahluwalia, and L. R. Collins, J. Fluid Mech. 536, 219 (2005).

[6] W. M. Durham, E. Climent, M. Barry, F. De Lillo, G. Boffetta, M. Cencini, and R. Stocker, Nat. Commun. 4, 2148 (2013).

[7] E. R. Abraham, Nature (London) 391, 577 (1998).

[8] F. De Lillo, M. Cencini, W. M. Durham, M. Barry, R. Stocker, E. Climent, and G. Boffetta, Phys. Rev. Lett. 112, 044502 (2014).

[9] K. Gustavsson, F. Berglund, P. R. Jonsson, and B. Mehlig, Phys. Rev. Lett. 116, 108104 (2016).

[10] R. E. Breier, C. C. Lalescu, D. Waas, M. Wilczek, and M. G. Mazza, Proc. Natl. Acad. Sci. U.S.A. 115, 12112 (2018).

[11] N. Peters, Turbulent Combustion, Cambridge Monographs on Mechanics (Cambridge University Press, Cambridge, England, 2000), https://doi.org/10.1017/CBO9780511612701.

[12] R. W. Bilger, Annu. Rev. Fluid Mech. 21, 101 (1989).

[13] G. Falkovich, A. Fouxon, and M. G. Stepanov, Nature (London) 419, 151 (2002).

[14] R. A. Shaw, Annu. Rev. Fluid Mech. 35, 183 (2003).

[15] J. Jucha, A. Naso, E. Lévêque, and A. Pumir, Phys. Rev. Fluids 3, 014604 (2018).

[16] E. Bodenschatz, S. P. Malinowski, R. A. Shaw, and F. Stratmann, Science 327, 970 (2010).

[17] G. A. Voth and A. Soldati, Annu. Rev. Fluid Mech. 49, 249 (2017).

[18] L. Chevillard and C. Meneveau, J. Fluid Mech. 737, 571 (2013). 
[19] S. Parsa, E. Calzavarini, F. Toschi, and G. A. Voth, Phys. Rev. Lett. 109, 134501 (2012).

[20] M. Byron, J. Einarsson, K. Gustavsson, G. Voth, B. Mehlig, and E. Variano, Phys. Fluids 27, 035101 (2015).

[21] N. R. Challabotla, L. Zhao, and H. I. Andersson, J. Fluid Mech. 766, R2 (2015).

[22] R. Ni, S. Kramel, N. T. Ouellette, and G. A. Voth, J. Fluid Mech. 766, 202 (2015).

[23] C. Meneveau, Annu. Rev. Fluid Mech. 43, 219 (2011).

[24] S. S. Girimaji and S. B. Pope, Phys. Fluids A 2, 242 (1990).

[25] M. Chertkov, A. Pumir, and B. I. Shraiman, Phys. Fluids 11, 2394 (1999).

[26] A. Naso and A. Pumir, Phys. Rev. E 72, 056318 (2005).

[27] L. Chevillard and C. Meneveau, Phys. Rev. Lett. 97, 174501 (2006).

[28] M. Wilczek and C. Meneveau, J. Fluid Mech. 756, 191 (2014).

[29] P. L. Johnson and C. Meneveau, J. Fluid Mech. 804, 387 (2016).

[30] L. Chevillard, C. Meneveau, L. Biferale, and F. Toschi, Phys. Fluids 20, 101504 (2008).

[31] P. L. Johnson and C. Meneveau, Phys. Rev. Fluids 2, 072601 (2017).

[32] P. L. Johnson and C. Meneveau, J. Fluid Mech. 837, 80 (2018).

[33] K. Ohkitani and S. Kishiba, Phys. Fluids 7, 411 (1995).

[34] C.-C. Wang, Arch. Rational Mech. Anal. 36, 166 (1970).

[35] G. Smith, Int. J. Eng. Sci. 9, 899 (1971).

[36] J. P. Boehler, Z. Angew. Math. Mech. 57, 323 (1977).

[37] S. Pennisi and M. Trovato, Int. J. Eng. Sci. 25, 1059 (1987).

[38] Q.-S. Zheng, Appl. Mech. Rev. 47, 545 (1994).

[39] Applications of Tensor Functions in Solid Mechanics, edited by J. P. Boehler, CISM Courses and Lectures No. 292 (Springer, Vienna, 1987), https://doi.org/10.1007/978-37091-2810-7.
[40] See Supplemental Material at http://link.aps.org/ supplemental/10.1103/PhysRevLett.125.224501 for details on the model derivation and the impact of the forcing amplitude, as well as details on the analysis of the FokkerPlanck equation and the simulations, which includes Refs. [41-46].

[41] A. J. M. Spencer and R. S. Rivlin, Arch. Rational Mech. Anal. 2, 309 (1958).

[42] A. J. M. Spencer and R. S. Rivlin, Arch. Rational Mech. Anal. 4, 214 (1959).

[43] S. B. Pope, J. Fluid Mech. 72, 331 (1975).

[44] P. Vieillefosse, J. Phys. France 43, 837 (1982).

[45] P. Vieillefosse, Physica (Amsterdam) 125A, 150 (1984).

[46] J. Martín, C. Dopazo, and L. Valiño, Phys. Fluids 10, 2012 (1998).

[47] J. Lawson and J. Dawson, J. Fluid Mech. 780, 60 (2015).

[48] R. Betchov, J. Fluid Mech. 1, 497 (1956).

[49] M. Carbone, M. Iovieno, and A. D. Bragg, J. Fluid Mech. 900, A38 (2020).

[50] P.E. Kloeden and E. Platen, Numerical Solution of Stochastic Differential Equations, Stochastic Modelling and Applied Probability Vol. 23 (Springer, Berlin, 1992), https://doi.org/10.1007/978-3-662-12616-5.

[51] P. A. Davidson, Turbulence: An Introduction for Scientists and Engineers (Oxford University Press, Oxford, New York, 2004).

[52] W. T. Ashurst, A. R. Kerstein, R. M. Kerr, and C. H. Gibson, Phys. Fluids 30, 2343 (1987).

[53] H. Xu, A. Pumir, and E. Bodenschatz, Nat. Phys. 7, 709 (2011).

[54] H. Yu and C. Meneveau, Phys. Rev. Lett. 104, 084502 (2010).

[55] G. B. Jeffery, Proc. R. Soc. A 102, 161 (1922).

[56] Y. Tian, D. Livescu, and M. Chertkov, in Physics Informed Learning of Lagrangian Turbulence: Velocity Gradient Tensor over Inertial-Range Geometry, 72nd Annual Meeting of the APS Division of Fluid Dynamics, Seattle, 2019, https://meetings.aps.org/Meeting/DFD19/Session/C17.3. 